ISSN 1991-8631

Original Paper

http://indexmedicus.afro.who.int

\title{
Inventaire de la faune mammalogique d'une forêt urbaine, le Parc National du Banco, Côte d'Ivoire
}

\author{
Eloi Anderson BITTY ${ }^{1,2 *}$, Blaise KADJO ${ }^{1}$, Sery GONEDELE BI ${ }^{2,3}$, \\ Modeste Okon OKON ${ }^{1}$ et Philippe Kouassi KOUASSI ${ }^{1}$ \\ ${ }^{1}$ Laboratoire de Zoologie et Biologie Animale, Université Félix Houphouët-Boigny, \\ Abidjan-Cocody, 22 BP 582 Abidjan 22, Côte d'Ivoire. \\ ${ }^{2}$ Centre Suisse de Recherche Scientifique en Côte d'Ivoire, 01 BP 1303 Abidjan 01, Côte d'Ivoire. \\ ${ }^{3}$ Laboratoire de Génétique, Université Félix Houphouët-Boigny, Abidjan-Cocody, Côte d'Ivoire, \\ 22 BP 582 Abidjan 22, Côte d'Ivoire. \\ *Auteur correspondant; E-mail : andersonbitty@yahoo.fr, eandersonbitty@gmail.com
}

\section{RESUME}

Le Parc National du Banco, forêt sempervirente à l'intérieure de la capitale économique de la Côte d'ivoire a toujours constitué une curiosité. Dans la période du 11 avril 2013 au 19 avril 2013 nous avons effectué dans cette forêt un inventaire des mammifères. Les méthodes utilisées sont celles des transects linéaires pour les grands mammifères et la méthode de capture pour la plupart des petits mammifères. Nos résultats indiquent que, les grands mammifères rencontrés sont constitués de primates (Cercopithecus campbelli lowei, Cercopithecus petaurista et Pan troglodytes verus), d'ongulés (Tragelaphus scriptus, Philantomba maxwellii, et Neotragus pygmaeus) et de carnivores (Genetta tigrina, Crossarchus obscurus et Civettictis civetta). D'une manière générale, l'indice kilométrique d'abondance de ces groupes taxonomiques est faible $(0,022$ à 0,12$)$. Le chimpanzé d'Afrique de l'Ouest (Pan troglodytes verus), espèce en danger selon l'Union pour la Conservation de la Nature existe encore dans cette forêt. Au niveau des petits mammifères plusieurs espèces de rongeurs, d'insectivores et de chiroptères ont été inventoriées lors de notre étude. Parmi ces espèces, la chauve-souris paillée (Eidolon helvum) et la Musaraigne de Wimmer (Crocidura wimmeri) sont en danger d'extinction selon l'UICN. Toutefois, la présence humaine à proximité du parc constitue une réelle menace pour son intégrité.

(ㄷ) 2013 International Formulae Group. All rights reserved.

Mots clés : Mammifères, chimpanzé, chauve-souris, insectivore.

\section{INTRODUCTION}

La zone forestière de la Côte d'Ivoire fait partie intégrante de la forêt de la haute Guinée, une zone de grande importance eu égard à sa grande diversité floristique et faunique (Myers, 2000 ; Kolongo et al., 2006). Malheureusement d'une manière générale et particulièrement en Côte d'Ivoire cette zone subit une pression anthropique consécutive à une croissance démographique galopante couplée d'une agriculture extensive et de l'exploitation forestière abusive. Rapidement la superficie forestière de la Côte d'Ivoire qui était de 16 millions d'hectares peu avant les indépendances de 1960 se situe actuellement à moins de quatre millions hectares (Brou Yao et al., 2005). Depuis, les forêts ivoiriennes ne cessent de subir de façon continuelle une diminution croissante. Au regard de cette fragmentation des forêts et de la disparition 
des espèces animales qu'elles abritent, les aires protégées (parcs nationaux et forêts classées) devraient jouer le rôle de refuge à ces populations animales. En effet, depuis 1926, la politique de conservation de la biodiversité, en Côte d'Ivoire est basée sur un réseau d'aires protégées réparties sur l'ensemble du territoire national (Koné, 2004). En 1987, on dénombrait 147 forêts classées d'une superficie totale de 2900000 hectares, huit parcs nationaux d'une superficie totale de 1732000 hectares et six réserves naturelles intégrales ou partielle d'une superficie de 339630 hectares (Koné, 2004). Toutefois, ces aires protégées se trouvent elles-mêmes fortement agressées pour la plupart d'entre elles. En outre, bien que la chasse soit interdite en Côte d'Ivoire depuis 1974 (arrêté 003/SEPN/CAB du 20 février 1974) cette activité illégale contribue à la disparition de nombreuses espèces à intérêt de conservation. Le Parc National du Banco (PNB) a été créé par Décret le 31 octobre 1953. Depuis sa création il reste un domaine relativement bien conservé. Ce parc, forêt dense a toujours constitué une curiosité et impressionne de part sa localisation au milieu des agglomérations de la ville d'Abidjan. Les études réalisées au PNB ont porté essentiellement sur les recensements botaniques (Kouamé et Zoro Bi, 2010) et celles relatives à son aménagement (Bassigny, 1995; Chautemps, 1998). Toutefois, très peu d'études ont été menées sur la faune (Lachenaud, 2006). La présence d'éléphants y avaient été mentionnés plusieurs décennies antérieures mais étaient en sursis à cette période et ont complètement disparu sous l'effet de la perte d'habitats connexes due à l'urbanisation accélérée de l'agglomération abidjanaise. En outre, des études plus récentes révèle le rôle de sanctuaire que joue ce parc pour plusieurs espèces de vertébrés (Kouadio, 2009 ; Kadjo et al., 2013). Malgré son importance écologique, le PNB fait l'objet d'agressions continuelles pour les prélèvements illicites des ressources végétales et animales. Par ailleurs, la pression foncière consécutive à la croissance démographique galopante de la ville d'Abidjan constitue une menace à la survie de cette forêt périurbaine. Cette étude a pour objectif d'évaluer la diversité des mammifères présents dans le parc et leur statut de conservation en vue de contribuer à sa meilleure conservation.

\section{MATERIEL ET METHODES \\ Site d'étude}

Le Parc National du Banco (PNB) couvre une superficie de 3474 hectares et est encerclé par l'agglomération d'Abidjan, capitale économique de la Côte d'Ivoire. Le PNB s'étend entre $5^{\circ} 21^{\prime}$ et $5^{\circ} 25^{\prime}$ de latitude Nord et $4^{\circ} 01^{\prime}$ et $4^{\circ} 05^{\prime}$ de longitude ouest (Figure 1). Il fut classé Réserve forestière (l'une des trois premières de Côte d'Ivoire) en 1926. Des travaux sylvicoles (reboisement de zones défrichées et plantations d'enrichissement) y ont alors été menés jusqu'en 1953, date de son classement en Parc National. Selon De Koning (1983) et Lauginie et al. (1996), le PNB est une forêt sempervirente. Les arbres dominants des strates supérieures sont Turraeanthus africanus, Synsepalum afzelii, Berlinia confusa, Blighia welwitschii, Coula edulis, Dacryodes klaineana, Lophira alata, Petersianthus macrocarpus, Piptadeniastrum africanum.

\section{Collecte des données}

Notre étude a été conduite de façon continue du 11 avril 2013 au 19 avril 2013.

\section{Technique d'inventaire des grands mammifères}

La méthode adoptée est celle des transects linéaires (Gonédélé et al., 2008 ; Gonédélé et al., 2012). Sept transects de 5 à $7,5 \mathrm{Km}$ chacun ont été placés à l'intérieur du parc. Les transects ont été positionnés de manière à prospecter les principaux habitats du site (Figure 2). Cette méthode d'échantillonnage consiste à marcher lentement le long des transects en vue de collectée des données relatives à la présence des mammifères. Les observations le long des transects peuvent être directes ou indirecte. $\mathrm{Au}$ total environ $50 \mathrm{~km}$ ont été parcourus pendant l'inventaire.

Les observations directes: elles concernent les individus de mammifères effectivement rencontrés durant l'étude.

Les observations indirectes : elles prennent en compte les indices de présence 
des mammifères (crottes, traces, latrines, nids, reste d'aliments, vocalisations ou cris etc.).

\section{Technique d'inventaire des petits mammifères}

Nous avons disposé des pièges de types Sherman et Longworth dans la forêt du Banco. La caractéristique de ces types de pièges est qu'ils permettent de capturer les animaux sans les tuer (Figure 3 ). Les pièges ont été disposés de manière à couvrir une grande partie du parc. Les coordonnées GPS (Global Positioning System) de tous les pièges ont été relevées de sorte à les retrouver toutes les fois que nous venions les visiter. Ces pièges sont visités tous les matins pendant la durée de l'étude. Pour tous les individus capturés nous avons relevé des données morphométriques discriminantes. Les chauves-souris ont été échantillonnées à l'aide de filets japonais de 12 mètres de long à mailles très fines de 20 × $20 \mathrm{~mm}$ (Figure 4). Quatre filets ont été déployés dans différents endroits du parc dans le but de maximaliser l'effort de capture. Ces filets ont été déployés de $18 \mathrm{~h} 00$ à $4 \mathrm{~h} 00$ et contrôlés toutes les 30 minutes. Les animaux capturés sont retirés des filets puis identifiés. En outre, toutes les observations directes de petits mammifères rencontrés sont relevées.

\section{Analyse des données}

De façon générale, le traitement des données a consisté aux calculs de fréquences en utilisant le logiciel Excel. Aussi avons nous calculé les indices kilométriques d'abondance en utilisant le logiciel Excel. Cet indice intègre le rapport entre les espèces rencontrées pendant la visite des transects et le nombre total de kilomètres parcourus.

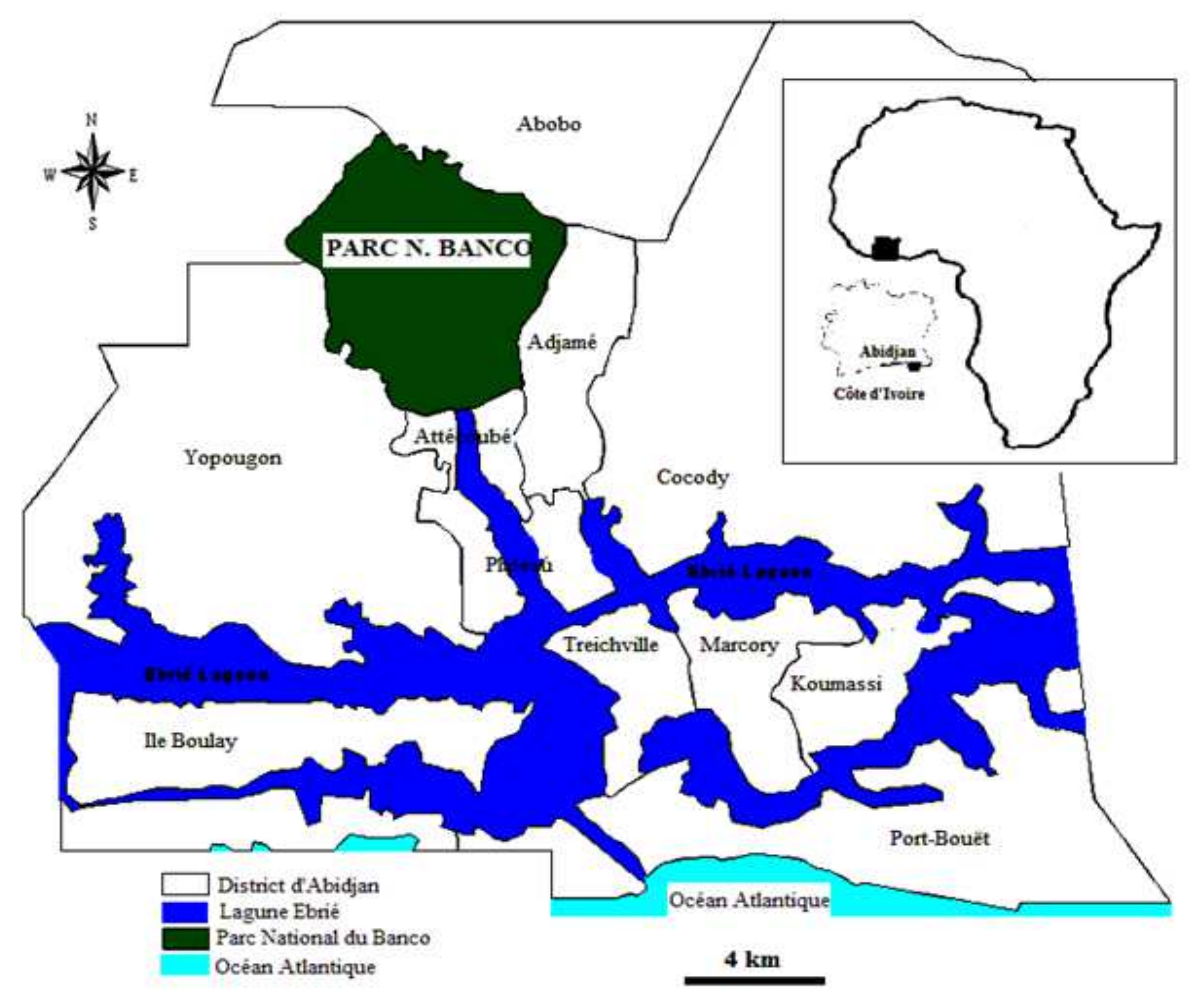

Figure 1 : Situation géographique du Parc National du Banco. 


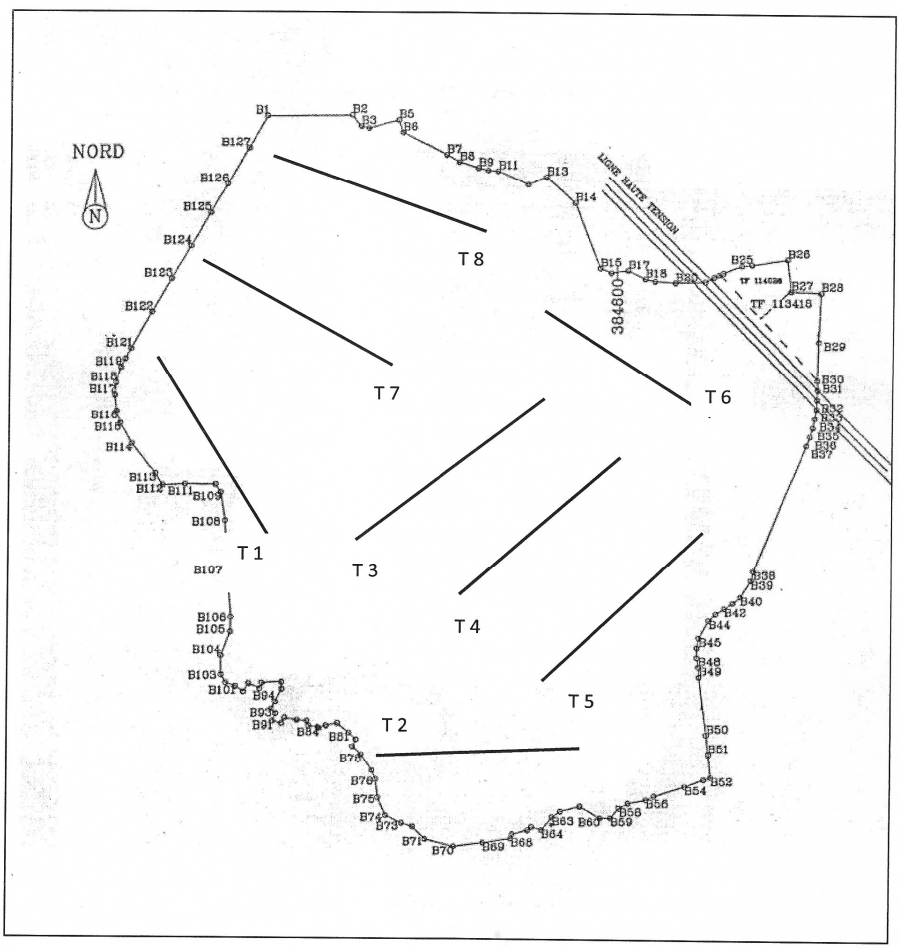

Figure 2 : Disposition des transects parcourus.

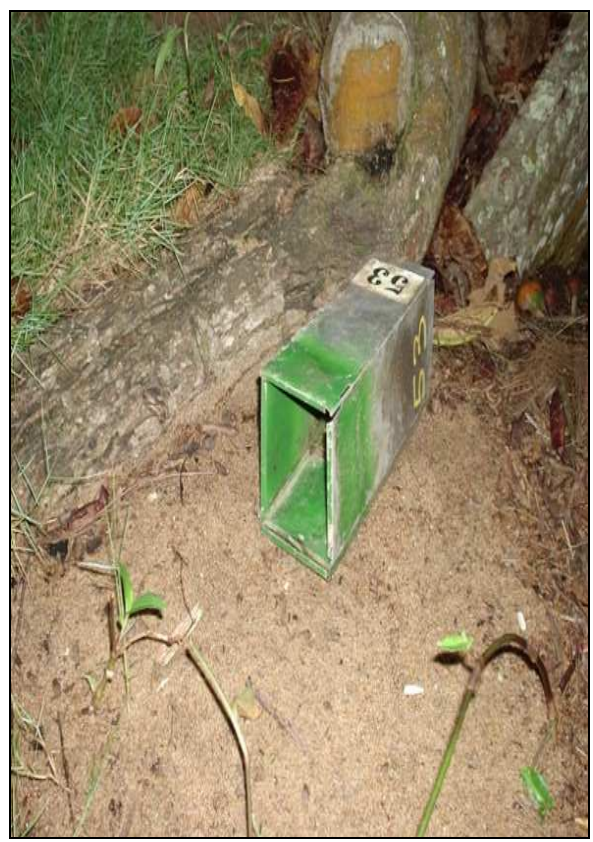

Piège de type Sherman

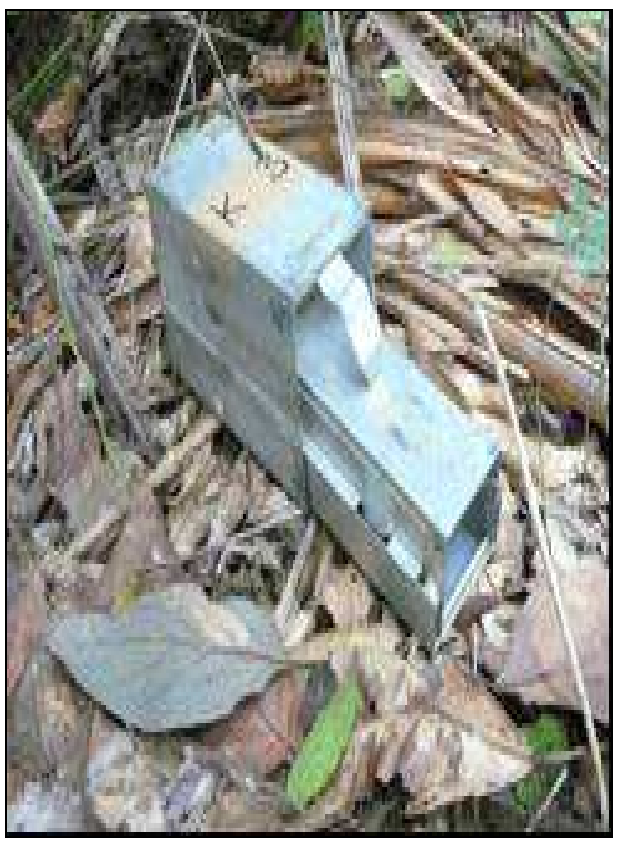

Piège de type Longworth

Figure 3 : Pièges de capture des petits mammifères. 


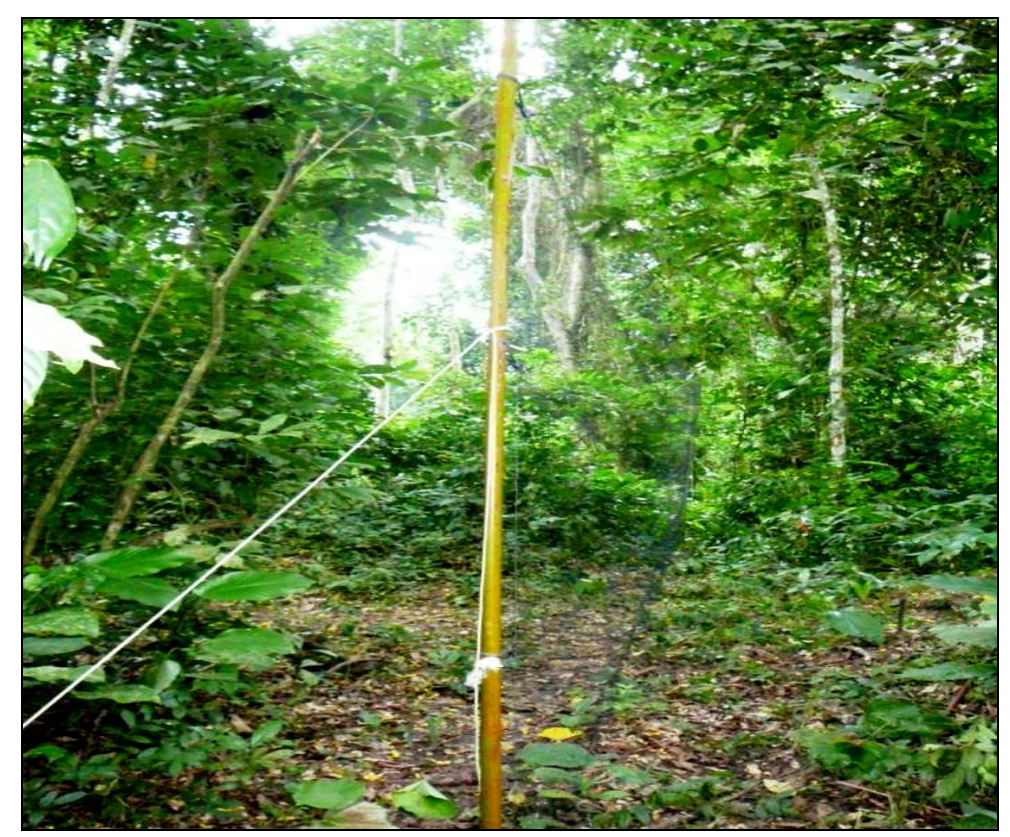

Figure 4 : Filet tendu pour la capture des Chauves-souris.

\section{RESULTATS}

\section{Grands mammifères}

De façon générale, les grands mammifères rencontrés au cours de notre étude sont constitués de primates (Cercopithecus campbelli lowei, Cercopithecus petaurista et Pan troglodytes verus), d'ongulés (Tragelaphus scriptus, Philantomba maxwellii, et Neotragus pygmaeus), de carnivores (Genetta tigrina, Crossarchus obscurus et Civettictis civetta). L'ensemble des observations qu'elles soient directes ou indirectes sont confinées dans le Tableau 1. Dans l'ensemble l'indice kilométrique d'abondance de ces groupes taxonomiques est relativement faible et vacille entre 0,02 et 0,12 . Le guib harnaché (Tragelaphus scriptus), les singes à queue (Cercopithecus campbelli lowei et Cercopithecus petaurista) et la mangouste commune (Crossarchus obscurus) sont les grands mammifères les plus couramment observés.

\section{Les petits mammifères}

Les petits mammifères sont représentés par les Insectivores (Musaraignes), les Rongeurs (souris, rats et mangoustes) et les Chiroptères ou Chauves-souris. Le Tableau 2 présente les communautés de petits mammifères capturés ou observés directement au cours de cette étude. Au total, 46 espèces de petits mammifères ont été capturées ou observés dont $45 \%$ appartiennent à l'ordre des Rongeurs, 39\% à l'ordre des Chiroptères (chauves-souris) et $16 \%$ à l'ordre des Insectivores (Figure 5). Les espèces dominantes de rongeurs sont Praomys tullbergi, Hylomyscus alleni et Lophuromys sikapusi. Au sein de la communauté de chauves-souris, la petite chauve-souris à épaulette (Epomops franqueti) est l'espèce dominante. Chez les insectivores deux espèces: Crocidura jouvenatae et Crocidura olivieri sont abondantes dans le groupe. 
Tableau 1 : Taux de rencontre des grands mammifères dans le Parc National du Banco et leur statut de conservation UICN (version 2011).

\begin{tabular}{|c|c|c|c|c|c|}
\hline \multirow[t]{2}{*}{ Nom commun } & \multirow[t]{2}{*}{ Nom scientifique } & \multirow{2}{*}{$\begin{array}{c}\begin{array}{c}\text { Nombre } \\
\text { d'observation (N) }\end{array} \\
\begin{array}{c}\text { Observation } \\
\text { directe }\end{array}\end{array}$} & \multirow{2}{*}{$\begin{array}{c}\mathrm{N} / \mathrm{Km} \\
\begin{array}{c}\text { Observation } \\
\text { indirecte }\end{array}\end{array}$} & \multicolumn{2}{|c|}{$\begin{array}{l}\text { Statut } \\
\text { UICN }\end{array}$} \\
\hline & & & & & \\
\hline \multicolumn{6}{|l|}{ PRIMATES } \\
\hline Hocheur nez-blanc & Cercopithecus petaurista & 3 & 2 & 0,1 & LC \\
\hline Mone de Campbell & Cercopithecus campbelli lowei & 3 & 2 & 0,1 & LC \\
\hline Chimpanzé & Pan troglodytes verus & 1 & - & 0,02 & EN \\
\hline Total primates & & 7 & 4 & & \\
\hline Guib harnaché & Tragelaphus scriptus & 1 & 5 & 0,12 & LC \\
\hline $\begin{array}{l}\text { Céphalophe de } \\
\text { Maxwell }\end{array}$ & Philantomba maxwellii & 1 & 2 & 0,06 & LC \\
\hline Antilope royale & Neotragus pygmaeus & 2 & - & 0,04 & LC \\
\hline Total ongulés & & 4 & 7 & & \\
\hline \multicolumn{6}{|l|}{ HYRACOIDES } \\
\hline Daman d'arbre & Dendrohyrax arboreus) & 1 & - & 0,02 & LC \\
\hline Total hyracoides & & 1 & & & \\
\hline \multicolumn{6}{|l|}{ CARNIVORES } \\
\hline Mangouste & Crossarchus obscurus & 3 & 2 & 0,1 & LC \\
\hline Civette d'Afrique & Civettictis civetta & - & 3 & 0,06 & LC \\
\hline Genette Tigrine & Genetta tigrina & - & 2 & 0,04 & LC \\
\hline Total carnivores & & 3 & 7 & & \\
\hline
\end{tabular}


Tableau 2 : Petits mammifères terrestres et volants vu ou capturés dans le Parc National du Banco.

\begin{tabular}{|c|c|c|c|}
\hline Taxon & Nom Français & $\begin{array}{l}\text { Nombre de } \\
\text { capture ou } \\
\text { présence }\end{array}$ & $\begin{array}{l}\text { Statut } \\
\text { UICN }\end{array}$ \\
\hline \multicolumn{4}{|l|}{ INSECTIVORES } \\
\hline Crocidura buettikoferi & Musaraigne noire & 1 & $\mathrm{LC}$ \\
\hline Crocidura jouvenetae & Musaraigne gracile & 5 & $\mathrm{LC}$ \\
\hline Crocidura muricauda & Musaraigne à queue de souris & 1 & $\mathrm{LC}$ \\
\hline Crocidura obscurior & Musaraigne naine & 1 & $\mathrm{LC}$ \\
\hline Crocidura olivieri & Musaraigne olivatre & 8 & $\mathrm{LC}$ \\
\hline Crocidura wimmeri & Musaraigne de Wimmer & 2 & EN \\
\hline Total Insectivores & & 20 & \\
\hline \multicolumn{4}{|c|}{ Nombre d'espèces $(S)=6$} \\
\hline \multicolumn{4}{|c|}{ CHIROPTERES (CHAUVES-SOURIS) } \\
\hline \multicolumn{4}{|c|}{ Mégachiroptères } \\
\hline Epomops buettikoferi & Grande Chauve-souris à épaulette & 8 & $\mathrm{LC}$ \\
\hline Eidolon helvum & Chauve-souris paillée & 1 & EN \\
\hline Hypsignathus monstrosus & Hypsignathe monstrueux & 2 & $\mathrm{LC}$ \\
\hline Megaloglossus azagnyi & Megaloglosse occidental & 13 & $\mathrm{LC}$ \\
\hline Myonycteris torquata & Chauve-souris à collier & 8 & $\mathrm{LC}$ \\
\hline Nanonycteris veldkampi & Microchauve-souris à épaulette & 2 & $\mathrm{LC}$ \\
\hline Scotonycteris zenkeri & Chauve-souris à spot facial & 1 & $\mathrm{LC}$ \\
\hline Total Mégachiroptères & & 49 & \\
\hline \multicolumn{4}{|l|}{ Nombre d'espèces $(S)=8$} \\
\hline Microchiroptères & & & \\
\hline Chaerephon sp. & Grand molosse & 1 & $\mathrm{LC}$ \\
\hline Epseticus sp. & Vespere & 2 & $\mathrm{LC}$ \\
\hline Hipposideros caffer & Chauve-souris à fer à cheval & 1 & $\mathrm{LC}$ \\
\hline Hipposideros cyclops & Chauve-souris à fer à cheval & 5 & $\mathrm{LC}$ \\
\hline Hipposideros ruber & Chauve-souris à fer à cheval & 2 & $\mathrm{LC}$ \\
\hline Mops condylurus & Molosse & 1 & $\mathrm{LC}$ \\
\hline Neoromicia nanulus & Pipistrelle naine & 2 & $\mathrm{LC}$ \\
\hline Nycteris grandis & Grand nyctère & 1 & $\mathrm{LC}$ \\
\hline Rhinolophus alcyone & Rhinolophe alcyone & 1 & $\mathrm{LC}$ \\
\hline Total Microchiroptères & & 16 & \\
\hline \multicolumn{4}{|l|}{ Nombre d'espèces $(S)=10$} \\
\hline \multicolumn{4}{|l|}{ Total Chiroptères $=17$ espèces } \\
\hline \multicolumn{4}{|l|}{ RONGEURS } \\
\hline Anomalurus beecrofti & Ecureil volant de Beecroft & $\mathrm{Vu}$ & $\mathrm{LC}$ \\
\hline Anomalurus peli & Ecureil volant de Pell & $\mathrm{Vu}$ & $\mathrm{LC}$ \\
\hline Atherurus africanus & Athérure & $\mathrm{Vu}$ & $\mathrm{LC}$ \\
\hline Cricetomys emini & Rat géant & 1 & $\mathrm{LC}$ \\
\hline Dephomys defua & Rat hérissé & 1 & $\mathrm{LC}$ \\
\hline Funisciurus pyrrhopus & Ecureuil & $\mathrm{Vu}$ & $\mathrm{LC}$ \\
\hline Graphiurus lorraineus & Graphiure naine & $\mathrm{Vu}$ & $\mathrm{LC}$ \\
\hline Heliosciurus rufobrachium & Ecureuil de soleil & $\mathrm{Vu}$ & $\mathrm{LC}$ \\
\hline Hybomys planifrons & Souris champêtre & 2 & $\mathrm{LC}$ \\
\hline Hybomys trivirgatus & Souris champêtre & 3 & $\mathrm{LC}$ \\
\hline Hylomyscus alleni & Souris champêtre & 7 & $\mathrm{LC}$ \\
\hline Lemniscomys striatus & Souris rayée & 2 & LC \\
\hline Lophuromys sikapusi & Souris rousse & 5 & $\mathrm{LC}$ \\
\hline Malacomys cansdelei & Souris à longue patte & 2 & $\mathrm{LC}$ \\
\hline
\end{tabular}




\begin{tabular}{llll}
\hline Malacomys edwardsi & Souris à longue patte & 5 & LC \\
Mastomys erythroleucus & Souris & 1 & LC \\
Mus musculö̈des & Souris & 1 & LC \\
Mus Nannomys setulosus & Souris naine & 1 & LC \\
Praomys rostratus & Souris champêtre & 6 & LC \\
Praomys tullbergi & Souris champêtre & 12 & LC \\
Protoxerus stangeri & Souris champêtre & $\mathrm{Vu}$ & LC \\
Thryonomys swinderianus & Aulacode & $\mathrm{Vu}$ & LC \\
Total Rongeurs & & $\mathbf{5 7}$ & \\
Nombre d'espèces $(\mathbf{S})=\mathbf{2 2}$ & & \\
\hline LC: Espèce d'intérêt de conservation mineur ; EN : Espèce en danger ; UICN : Union International pour la Conservation \\
de la Nature.
\end{tabular}

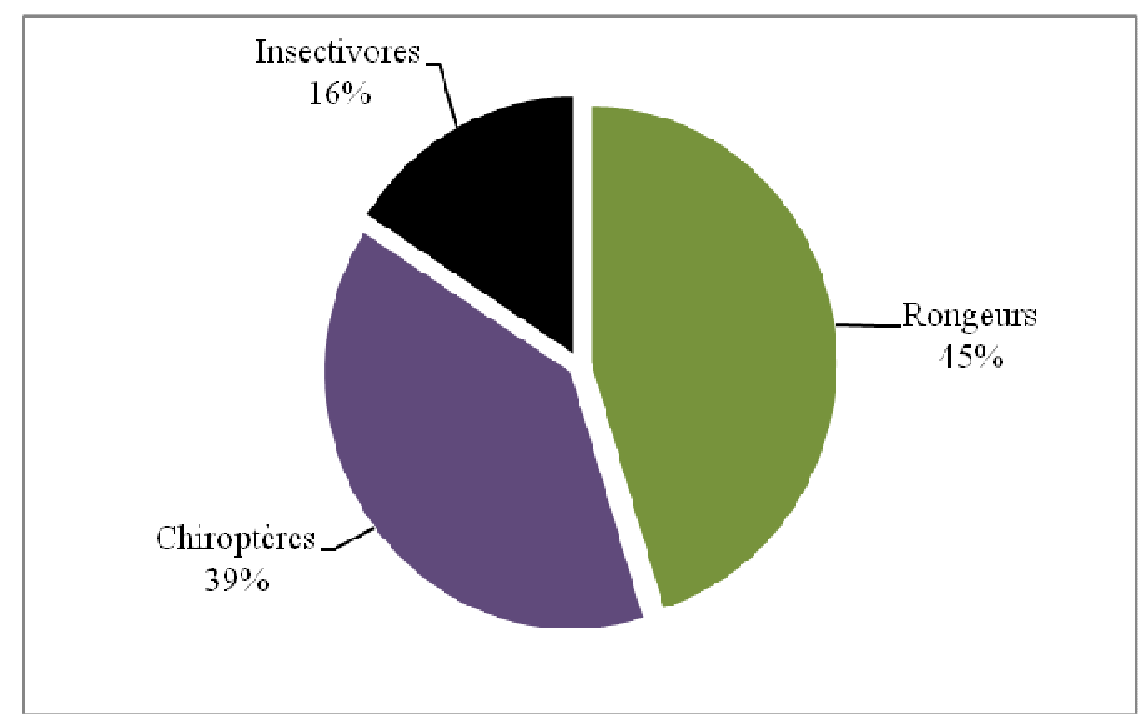

Figure 5 : Répartition des petits mammifères inventoriés.

\section{DISCUSSION}

Bien que les indices kilométriques d'abondance des grands mammifères observés durant notre étude soient faibles, nos résultats révèlent que le Parc National du Banco abrite encore plusieurs espèces de grands mammifères. Les ongulés (guib harnachés) et les primates constituent les grands mammifères les plus fréquemment observés. A l'exception du chimpanzé, tous les grands mammifères observés sont d'une préoccupation mineure de conservation (IUCN, 2011). Toutefois, ces animaux présentent un intérêt dans la préservation de la biodiversité et jouent un rôle primordial dans le maintien et l'équilibre des réseaux trophiques. Le chimpanzé d'Afrique de l'Ouest (Pan troglodytes verus), espèce emblématique vit toujours dans le Parc National du Banco alors que cette espèce a disparu de la plupart des forêts de Côte d'Ivoire et de la sous-région. Le chimpanzé (Pan troglodytes verus) demeure une espèce à grand intérêt de conservation eu égard à sa disparition rapide et brutale de son aire originelle de distribution ce qui lui a valu d'être classé sur la liste rouge des espèces gravement menacées d'extinction de l'Union International pour la Conservation de la Nature (IUCN, 2011). En effet, selon des études antérieures, le Parc National de Taï en Côte d'Ivoire apparaît comme l'une des rares 
forêts à avoir une population de chimpanzé suffisamment grande pour permettre le maintien de cette espèce dans le pays (Marchesi et al., 1995). Toutefois, la seule présence d'une communauté de chimpanzés à l'état sauvage dans une forêt périurbaine témoigne de son état de conservation. Raison pour laquelle il est plus qu'urgent d'œuvrer à la préservation de cette relique de forêt. Malheureusement, le Parc National du Banco est soumis à une forte pression anthropique consécutive à la proximité des habitations et à une pression intense de braconnage (Lauginie et al., 1996; Lachenaud, 2006). Ceci expliquerait les faibles indices kilométriques d'abondance des grands mammifères et de leur faible diversité. L'abondance relative du guib harnaché, commensale de l'homme pourrait témoigner de l'importance de l'anthropisation du parc. En effet, la pression anthropique est très perceptible au niveau de la limite nord du parc, soumise à une pression d'occupation humaine.

Par ailleurs, le parc héberge une diversité exceptionnelle de petits mammifères parmi lesquels la chauve-souris paillée (Eidolon helvum) et la Musaraigne de Wimmer (Crocidura wimmeri), deux espèces en danger d'extinction et classées sur la liste rouge de l'UICN (IUCN, 2011). En effet, outre leur rôle essentiel dans l'équilibre des réseaux trophique, La gestion du patrimoine naturel exige en priorité la prise en compte de ses composantes les plus sensibles. La présence de certains petits mammifères tel que Crocidura wimmeri est un indicateur de l'état de conservation de milieu naturel eu égard leur inféodation aux habitats particuliers (Decher et al., 2005). Hormis C. wimmeri, la plupart des espèces de rongeurs et des insectivores capturés sont des espèces communes dont certaines peuvent même être rencontrés dans les milieux anthropisés (paysages agricoles). Bien que la faune de Chiroptères renferme plus d'espèces de Microchiroptères que de Mégachiroptères, inversement le nombre d'individus de Mégachiroptères est le triple de celui des Microchiroptères. Le faible taux de capture de microchiroptères s'explique par le fait qu'ils détectent la présence des filets sur leur passage grâce à leur système d'écholocation
(Britton et Jones, 1999). Lorsqu'ils sont pris dans les filets, ils découpent assez rapidement les filets grâce à leurs dents acérées pour s'en échapper (Decher et al., 2005).

\section{Conclusion}

Le Parc National du Banco abrite plusieurs espèces de mammifères reparties en plusieurs ordres: les Ongulés, les Primates, les Carnivores, les Hyracoides, les Insectivores, les Chiroptères et les Rongeurs. Parmi ces mammifères vivant dans le parc trois d'entre eux, le chimpanzé (Pan troglodytes verus), la chauve-souris paillée (Eidolon helvum) et la Musaraigne de Wimmer (Crocidura wimmeri) sont gravement menacés d'extinction et figurent sur la liste rouge de l'UICN. La présence de ces espèces emblématiques dans la forêt du banco constitue un indicateur de l'importance de cette forêt périurbaine et de la nécessité de la conserver durablement. Toutefois, cette relative diversité faunique du parc est entamée par une pression humaine très intense relative à la proximité des habitations. Cette présence humaine constitue une réelle menace pour l'intégrité du parc et un défi pour les gestionnaires de ce patrimoine pour sa préservation.

\section{REMERCIEMENTS}

Nous remercions la Direction Générale de l'OIPR (Office Ivoirien des Parcs et Réserves) de nous avoir accordé l'autorisation d'accès au Parc National du Banco sans laquelle cette étude n'aurait pas été possible. Nous exprimons notre reconnaissance au Capitaine Bomisso Germain, Chef-Secteur du Parc National du Banco pour son assistance technique durant les inventaires. Enfin nous remercions Monsieur Hilaire, notre guide pour son aide pendant les inventaires.

\section{REFERENCES}

Bassigny JF, Beligné V, Lauginie F. 1995. Conservation du Parc national du Banco : étude de l'état du milieu naturel et recommandations pour sa sauvegarde; étude d'avant-projet des infrastructures. WWF, p. 48.

Britton ARC, Jones G. 1999. Echolocation behaviour and prey capture success in foraging bats: laboratory and field 
experiments on Myotis daubentonii. $J$. Exp. Biol., 202: 1793-1801.

Brou Yao T, Oszwald J, Bigot S, Dervat E. 2005. Risques de Déforestation dans le Domaine Permanent de l'Etat de Côte d'Ivoire: Quel Avenir pour ces Derniers massif Forestiers ? Télédétection., 5(1): 205-121.

Chautemps V. 1998. Perspectives d'aménagement et de protection du Parc national du Banco. Mémoire de Maîtrise des Sciences et Techniques d'Aménagement, Institut de Géographie et d'Aménagement. Université de Nantes ; France, p. 116.

De Koning J. 1983. La forêt du Banco. Thèse de Doctorat de l'Université de Wageningen, Pays-Bas, volumes I et II, p. 1500 .

Decher J, Kadjo B, Abedi ML, Elhadji OT, Soumaoro K. 2005. Une étude rapide des petits mammifères (musaraignes, rongeurs et chiroptères) des forêts classées de la haute Dodo et du Cavally, Côte d'Ivoire. In $R A P \quad$ Bulletin $N^{\circ}$ 34: $A$ Rapid Biological Assessment of Two Classified Forests in South-Western Côte d'Ivoire, Lauginie F, Rondeau G, Alonso LE (eds). Conservation International: Washington, D.C; 101-109.

Gonédélé BS, Koné I, Béné KJC, Bitty EA,Akpatou KB, Gone BZB, Ouattara K, Koffi DA. 2008. Tanoé forest, southeastern Côte-d'Ivoire identified as a high priority site for the conservation of critically endangered Primates in West Africa. Trop. Cons. Sci, 1(3): 265-278.

Gonédélé BS, Koné I, Béné KJ-C, Bitty EA, Koffi BJC, Akpatou B, Zinner D. 2012. Distribution and Conservation Statusof Catarrhine Primates in Côte d'Ivoire (West Africa). Folia Primatol., 83: 11-23.

IUCN 2011. IUCN Red List of Threatened Species. Version 2011.2. www. iucnredlist.org

Kadjo B, Kouadio YR, Vogel V, Dubey S, Vogel P. 2013. Assessment of terrestrial small mammals with record of the endangered shrew Crocidura wimmeri Heim de Balsac \& Aellen 1958 in Banco National Park (Côte d'Ivoire). Mammalia (Sous Press).
Kolongo TSD, Decocq G, Yao CYA, Blom EC, Rompaey RSA. 2006. Plant species diversity in the southern part of the Tai National Park (Côte d'Ivoire). Biodiversity and Conservation, 15: 21232142.

Koné I. 2004. Effet du braconnage sur quelques aspects du comportement du colobe bai Procolobus [piliocolobus] badius et $\mathrm{du}$ cercopitèque diane Cercopithecus diana diana dans le Parc National de Taï, Côte d'Ivoire, Thèse de Doctorat, Université de Cocody, Abidjan, p. 146.

Kouadio YR. 2009. Diversité des rongeurs et insectivores du Parc National du Banco. Mémoire de DEA. Université de Cocody, Côte d'Ivoire, p. 76.

Kouamé NF, Zoro Bi IA. 2010. Nouveau découpage de la zone de forêt dense humide de la Côte d'Ivoire. Science \& Nature., 7(2) : 177-194.

Lachenaud O. 2006. Les oiseaux du Parc National du Banco et de la Forêt Classée de l'Anguédédou. Malimbus., 28: 107132.

Lauginie F, Poilecot P, Akindes F, Béligné V, Bonfou K, Ouattara D. 1996. Monographie des Parcs Nationaux. Document 5. Projet WWF - CI008 Revue générale des parcs nationaux et réserves de Côte d'Ivoire, p. 137.

Marchesi P, Marchesi N, Fruth B, Boesch C. 1995. Census and distribution of chimpanzees in Côte d'Ivoire. Primate., 36: 591-607.

Myers M, Mittermeier RA, Mittermeier CG, Da Fonseca GAB, Kent J. 2000. Biodiversity hotspots for conservationpriorities. Nature, 403: 853858. 